\title{
Time-reversal symmetry violation in several Lepton-Flavor-Violating processes
}

\author{
Juan Carlos Vasquez \\ ICTP, \\ Strada Costiera, 11, 34151 Trieste, Italy \\ SISSA/INFN, \\ via Bonomea, 265, 34136 Trieste, Italy \\ Gran Sasso Science Institute, \\ Viale Crispi 7, 67100 L'Aquila, Italy \\ E-mail: jcvasque@sissa.it
}

ABSTRACT: We compute a T-odd triple vector correlation for the $\mu \rightarrow e \gamma$ decay and the $\mu \rightarrow e$ conversion process, finding simple results in terms of the CP violating phases of the effective Hamiltonians. Then we focus on the minimal Left-Right symmetric extension of the Standard Model, which can lead to an appreciable correlation. We show that under rather general assumptions, this correlation can be used to discriminate between Parity or Charge-conjugation as the discrete Left-Right symmetry.

Keywords: Rare Decays, Beyond Standard Model, Neutrino Physics, CP violation

ARXIV EPRINT: 1504.05220 


\section{Contents}

1 Introduction 1

2 General theory 2

$2.1 \mu \rightarrow e \gamma$ process 2

$2.2 \mu \rightarrow e$ conversion. Theory and effective Hamiltonian 3

3 The minimal Left-Right symmetric theory 5

4 Computation of a triple vector correlation in the $\mu \rightarrow e \gamma$ decay $\quad 7$

5 Computation of a triple vector correlation in the $\mu \rightarrow e$ conversion process

6 Triplet vector correlation in the minimal Left-Right theory 9

$6.1 \mu \rightarrow e \gamma$ decay 9

$6.2 \mu \rightarrow e$ conversion process 13

$\begin{array}{lll}7 & \text { Conclusions } & 15\end{array}$

A Kinematics of the $\mu \rightarrow e \gamma$ process and the triple vector correlation $\quad 15$

B $\mu \rightarrow e$ total conversion rate and the triple vector correlation 16

$\begin{array}{ll}\text { B.1 Total conversion rate } & 16\end{array}$

$\begin{array}{ll}\text { B.2 Triple vector correlation in the conversion process } & 17\end{array}$

\section{Introduction}

Lepton Number Violating (LNV) and Lepton Flavor violating (LFV) processes are forbidden in the Standard Model (SM) and are thus a good probe of new physics. In principle new physics brings also new sources of $\mathrm{CP}$ violation and therefore time reversal $(\mathrm{T})$ symmetry violation in any local, Lorentz invariant quantum field theory.

Motivated by this we explicitly compute T-odd triple vector correlations for the LFV $\mu \rightarrow e \gamma$ decay and $\mu \rightarrow e$ conversion process, since much of the present and future experimental efforts are devoted to these two processes. The MEG collaboration reports the best experimental limit for the $\mu \rightarrow e \gamma$ decay [1]

$$
\operatorname{Br}(\mu \rightarrow e \gamma) \equiv \frac{\Gamma(\mu \rightarrow e \gamma)}{\Gamma\left(\mu \rightarrow e \nu_{\mu} \nu_{e}\right)}<5.7 \times 10^{-13}
$$


and the SINDRUM II collaboration gives the strongest limits for the $\mu \rightarrow e$ conversion process $[2,3]$, namely

$$
\mathbf{B r}(\mu+\operatorname{Ti}(\mathrm{Au})) \rightarrow e+\operatorname{Ti}(\mathrm{Au})) \equiv \frac{\Gamma(\mu \rightarrow e)}{\Gamma_{\text {capt }}}<6.1(7) \times 10^{-13},
$$

where $\Gamma_{\text {capt }}$ is the muon capture rate in the vicinity of a nucleus. Upgrades of ongoing experiments have been considered with the final goal of achieving a sensitivity around $10^{-18}-10^{-19}[4-7]$. Given the current limits and the future improvements, there exist the possibility of having enough statistics to start probing $\mathrm{CP}$ violation beyond the SM in the next round of experiments. This is suggested and studied in $[8,9]$.

In this work we focus on quantities that test $\mathrm{T}$ violation in the absence of finalstate interactions and among these quantities are triple vector correlations made up of the momenta or spins of the participating particles [10]. In [11], it is suggested that triplet vector correlations can be used to probe $\mathrm{CP}$ violation in the $\mu \rightarrow e$ conversion process. Here we present the first analytical computation for the correlation suggested in [11] for the $\mu \rightarrow e$ conversion process and we extend their work in two ways: first, we compute the correlation for the $\mu \rightarrow e \gamma$ decay and second we include the full set of effective operators that enter the $\mu \rightarrow e$ conversion process.

In section 2 we introduce some theoretical tools for the $\mu \rightarrow e \gamma$ and the $\mu \rightarrow e$ conversion process. In section 3 as an example of a theory that gives order one contribution to the triple vector correlation, we briefly introduce the minimal Left-Right (LR) symmetric extension of the SM. In section 4 and 5 we present the analytical computation of the triple correlation in the $\mu \rightarrow e \gamma$ and $\mu \rightarrow e$ conversion process respectively. Then in section 6 and for both processes, we study these correlations in the context of the minimal LR model, for both parity and charge-conjugation as the LR symmetries. Finally in section 7 we present our conclusions.

\section{General theory}

\section{$2.1 \mu \rightarrow e \gamma$ process}

The $\mu \rightarrow e \gamma$ decay is predicted to be negligible small in the SM with massive neutrinos, therefore if this process is seen it implies that new physics is behind it. The effective Hamiltonian for this process is of the form

$$
H_{\mathrm{eff}}=\frac{4 e G_{F} m_{\mu}}{\sqrt{2}} \bar{e}\left(p_{e}\right) \sigma_{\mu \nu} F^{\mu \nu}\left(A_{L} P_{L}+A_{R} P_{R}\right) \mu\left(p_{\mu}\right)+h . c .,
$$

where $e$ is the electromagnetic coupling constant, $F_{\mu \nu}$ is the electromagnetic field strength for the photon field, $G_{F}$ is the Fermi constant, $P_{(R, L)} \equiv \frac{1}{2}\left(1 \pm \gamma_{5}\right), m_{\mu}$ is the muon mass and $e\left(p_{e}\right)$ and $\mu\left(p_{\mu}\right)$ are the spinors for the electron and muon respectively. For this process we use the gamma matrices in the Weyl basis and the coefficients $A_{L}$ and $A_{R}$ are calculated within a given physical model. 


\section{2 $\mu \rightarrow e$ conversion. Theory and effective Hamiltonian}

Theoretical studies of this process were performed in the past [12-15]. In [15] the outgoing electron coming from the conversion process, belongs to one of the states in the continuum energy spectrum for the Coulomb potential and as a matter of fact the outgoing electron must be treated as a plane wave. One way to argue this is by noticing that an electron in the continuum energy spectrum, is described by a Dirac spinor in the angular momentum basis. Experimentally, the detected electron has a define 4-momentum implying that the outgoing electron must be a plane wave.

In this work we present a method for computing a triple vector correlation that tests Tviolation in the $\mu \rightarrow e$ conversion process for various nuclei. We make use of the formalism developed in [16].

We use the following representation for the $\gamma$ matrices

$$
\gamma_{0}=\beta=\left(\begin{array}{cc}
1 & 0 \\
0 & -1
\end{array}\right), \quad \gamma_{i}=\left(\begin{array}{cc}
0 & \sigma_{i} \\
-\sigma_{i} & 0
\end{array}\right)
$$

and

$$
\sigma_{\mu \nu}=\frac{i}{2}\left[\gamma_{\mu}, \gamma_{\nu}\right], \quad \gamma_{5}=-i \gamma_{1} \gamma_{2} \gamma_{3} \gamma_{0}
$$

where the $\sigma_{i}$ are the Pauli matrices where $i=1,2,3$ and the index $\mu$ takes the values $\mu=0,1,2,3$.

The Dirac's equation for the central field problem in polar coordinates is given by (the energy is given in units of the electron mass)

$$
E \psi=H \psi=\left[-i \gamma_{5} \Sigma_{r}\left(\frac{\partial}{\partial r}+\frac{1}{r}-\frac{\beta}{r} K\right)+V+\beta\right] \psi,
$$

where

$$
\begin{aligned}
\Sigma_{r} & =\frac{1}{r} \sum_{i} \Sigma_{i}, \quad \Sigma_{i}=\frac{i}{2}\left[\gamma_{j}, \gamma_{k}\right] \quad(\{\mathrm{i}, \mathrm{j}, \mathrm{k}\} \text { cyclic }) . \\
K & =\beta(\Sigma \cdot L+1) .
\end{aligned}
$$

$V$ is the Coulomb potential and $L$ is the orbital angular momentum.

We write the wave function as [17]

$$
\psi_{\kappa}^{\mu}=\left(\begin{array}{c}
g_{\kappa}(r) \chi_{\kappa}^{\mu} \\
i f_{\kappa}(r) \chi_{-\kappa}^{\mu}
\end{array}\right),
$$

such that $K \psi_{\kappa}^{\mu}=-\kappa \psi_{\kappa}^{\mu}$ and $J_{3} \psi_{\kappa}^{\mu}=\mu \psi_{\kappa}^{\mu}$, where $J_{3}$ is the third component of the total angular momentum $\vec{J}$. The radial functions $g_{\kappa}$ and $f_{\kappa}$ obey the differential equations

$$
\begin{aligned}
\frac{d g_{\kappa}(r)}{d r} & =-\frac{\kappa+1}{r} g_{\kappa}(r)+(E-V+1) f_{\kappa}(r), \\
\frac{d f_{\kappa}(r)}{d r} & =\frac{\kappa-1}{r} f_{\kappa}(r)-(E-V-1) g_{\kappa}(r) .
\end{aligned}
$$


In the high energy limit -all the masses are set to zero- and from eqs.(2.8) and (2.9), $f_{\kappa}(r)$ and $g_{\kappa}(r)$ satisfy

$$
f_{-\kappa}=-g_{\kappa}, \quad g_{-\kappa}=f_{\kappa} .
$$

From here on we make use of this result for the spinor $\psi_{\kappa, E}^{\mu(e)}$ describing the electrons coming from the conversion process. The initial muon instead is described by $\psi_{\kappa}^{\mu}$ with the quantum numbers, $\mu= \pm \frac{1}{2}$ and $\kappa=-1$ and we choose the normalization

$$
\int d^{3} x \psi_{1 s}^{(\mu) \dagger}(\vec{x}) \psi_{1 s}^{(\mu)}(\vec{x})=1
$$

For the electrons in the continuum-energy states we use the same normalization considered in [15], namely

$$
\int d^{3} x \psi_{\kappa, E}^{\mu(e) \dagger}(\vec{x}) \psi_{\kappa^{\prime}, E^{\prime}}^{\mu^{\prime}(e)}(\vec{x})=2 \pi \delta_{\mu \mu^{\prime}} \delta_{\kappa^{\prime} \kappa} \delta\left(E-E^{\prime}\right) .
$$

In the conversion process the effective Hamiltonian is given by [15]

$$
\begin{aligned}
H_{\mathrm{eff}}= & \frac{4 G_{F}}{\sqrt{2}}\left(m_{\mu} A_{R}^{*} \bar{\mu} \sigma^{\mu \nu} P_{L} e F_{\mu \nu}+m_{\mu} A_{L}^{*} \bar{\mu} \sigma^{\mu \nu} P_{R} e F_{\mu \nu}+\text { h.c. }\right) \\
& +\frac{G_{F}}{\sqrt{2}} \sum_{q=u, d, s}\left[\left(g_{L S(q)} \bar{e} P_{R} \mu+g_{R S(q)} \bar{e} P_{L} \mu\right) \bar{q} q+\left(g_{L P(q)} \bar{e} P_{R} \mu+g_{R P(q)} \bar{e} P_{L} \mu\right) \bar{q} \gamma_{5} q\right. \\
& \left(g_{L V(q)} \bar{e} \gamma^{\mu} P_{L} \mu+g_{R V(q)} \bar{e} \gamma^{\mu} P_{R} \mu\right) \bar{q} \gamma_{\mu} q+\left(g_{L A(q)} \bar{e} \gamma^{\mu} P_{L} \mu+g_{R A(q)} \bar{e} \gamma^{\mu} P_{R} \mu\right) \bar{q} \gamma_{\mu} \gamma_{5} q \\
& \left.+\frac{1}{2}\left(g_{L T(q)} \bar{e} \sigma^{\mu \nu} P_{R} \mu+g_{R T(q)} \bar{e} \sigma^{\mu \nu} P_{L} \mu\right) \bar{q} \sigma_{\mu \nu} q+\text { h.c. }\right] .
\end{aligned}
$$

The nuclear form factors were calculated in [18]. The wave function for the muon and the electrons in the presence of a central field were obtained in $[14,15]$. In particular in [15] updated data for the proton and neutron densities were used.

In the limit of $r \rightarrow \infty$ it can be shown that the general solution for a Dirac particle in a Coulomb field at first order in $H_{\text {eff }}$ is of the form [16]

$$
\psi_{a s}=-i \sqrt{\frac{\pi}{\mid \vec{p}} \mid} \frac{e^{i p r}}{r} \sum_{\kappa \mu} e^{i \delta_{\kappa}}\left\langle\psi_{\kappa}^{(e) \mu}\left|H_{\mathrm{eff}}\right| \psi_{1 s}^{(\mu)}\right\rangle\left(\begin{array}{c}
\sqrt{E+1} \chi_{\kappa}^{\mu}(\hat{p}) \\
-\sqrt{E-1} \chi_{-\kappa}^{\mu}(\hat{p})
\end{array}\right)+\mathcal{O}\left(H_{\mathrm{eff}}^{2}\right),
$$

where $\hat{p}$ is in the direction of the outgoing electron. The phases $e^{i \delta_{\kappa}}$ are the usual ones appearing in scattering problems in the presence of a Coulomb field and are given by

$$
\begin{aligned}
\delta_{\kappa} & =y \ln 2 p r-\arg \Gamma(\gamma+i y)+\eta_{\kappa}-\frac{1}{2} \pi \gamma, \\
y & =\alpha Z E / p, \quad \gamma=\sqrt{\kappa^{2}-\alpha^{2} Z^{2}}, \quad e^{2 i \eta_{\kappa}}=-\frac{\kappa-i y / E}{\gamma+i y}
\end{aligned}
$$

where $Z$ is the atomic number, $\alpha=e^{2} / 4 \pi$ and $p$ is the modulus of the 3 -momentum $\vec{p}$. We consider states with $\kappa= \pm 1$, hence the only term relevant for our discussion is $\eta_{\kappa}$-the remaining ones are just an overall phase in the solution $\psi_{a s}$. 
Finally the total conversion rate per unit flux is ${ }^{1}$

$$
\omega_{\text {conv }}=R^{2} \int d \Omega \psi_{a s}^{\dagger} \psi_{a s}=\frac{1}{2} \sum_{\kappa, \mu}\left|\left\langle\psi_{\kappa}^{\mu}\left|H_{\text {eff }}\right| \psi_{i}\right\rangle\right|^{2} .
$$

\section{The minimal Left-Right symmetric theory}

As an example of a complete and predictive theory of lepton number violating phenomena we consider the minimal LR symmetric extension of the SM [19-22]. In this model the gauge group is $\mathrm{SU}(2)_{L} \times \mathrm{SU}(2)_{R} \times \mathrm{U}(1)_{B-L}$ with an additional discrete symmetry that may be generalized parity $(P)$ or charge conjugation $(C)$-for reviews see [23-25]. It relates the smallness of neutrino masses to the near maximality of parity violation al low energies through the seesaw mechanism [27-32]. The scalar sector contains the following fields [26-29]

$$
\Phi=\left(\begin{array}{cc}
\phi_{1}^{0} & \phi_{2}^{+} \\
\phi_{1}^{-} & \phi_{2}^{0}
\end{array}\right), \quad \Delta_{L, R}=\left(\begin{array}{cc}
\delta_{L, R}^{+} / \sqrt{2} & \delta_{L, R}^{++} \\
\delta_{L, R}^{0} & -\delta_{L, R}^{+} / \sqrt{2}
\end{array}\right)
$$

where $\Phi$ is in the $(2,2,0)$ representation of $\mathrm{SU}(2)_{L} \times \mathrm{SU}(2)_{R} \times \mathrm{U}(1)_{B-L}$ and the two scalar triplets $\Delta_{L}$ and $\Delta_{R}$, belong to the $(3,1,2)$ and the $(1,3,2)$ representations respectively. The Yukawa interactions of leptons with the scalar triplets have the form

$$
\mathcal{L}_{Y}=\bar{L}_{L}\left(Y_{\Phi} \Phi+\tilde{Y}_{\Phi} \tilde{\Phi}\right) L_{R}+\frac{1}{2}\left(L_{L}^{T} C i \sigma_{2} Y_{\Delta_{L}} \Delta_{L} L_{L}+L_{R}^{T} C i \sigma_{2} Y_{\Delta_{R}} \Delta_{R} L_{R}\right)+\text { h.c. },
$$

$\tilde{\Phi}=\sigma_{2} \Phi^{*} \sigma_{2}, L_{L}$ is the lepton doublet of the standard model $\left(L_{L}^{T}=\left(\begin{array}{ll}\nu & l\end{array}\right)_{L}\right)$ and $L_{R}$ is its right-handed analogue that we denote as $L_{R}^{T}=\left(\begin{array}{lll}N & l\end{array}\right)_{R}$ where $N$ is the heavy Majorana neutrino. The $Y_{a}$ is the Yukawa coupling of the field $a$, where $a=\left\{\Phi, \tilde{\Phi}, \Delta_{L}, \Delta_{R}\right\}$.

Under the discrete left-right symmetry the fields of the theory transform as:

$$
P:\left\{\begin{array}{l}
\mathcal{P} f_{L} \mathcal{P}^{-1}=\gamma_{0} f_{R} \\
\mathcal{P} \Phi \mathcal{P}^{-1}=\Phi^{\dagger} \\
\mathcal{P} \Delta_{L, R} \mathcal{P}^{-1}=-\Delta_{R, L}
\end{array} \quad, \quad C:\left\{\begin{array}{l}
\mathcal{C} f_{L} \mathcal{C}^{-1}=\boldsymbol{C}\left(\overline{f_{R}}\right)^{T} \\
\mathcal{C} \Phi \mathcal{C}^{-1}=\Phi^{T} \\
\mathcal{C} \Delta_{L, R} \mathcal{C}^{-1}=-\Delta_{R, L}^{*}
\end{array}\right.\right.
$$

where the usual charge conjugation operator is given by $\boldsymbol{C}=i \gamma_{2} \gamma_{0}$.

Invariance of the Lagrangian under the LR symmetry leads to the following relations between the Yukawa couplings of the theory, namely

$$
P:\left\{\begin{array}{l}
Y_{\Delta_{R, L}}=Y_{\Delta_{L}, R} \\
Y_{\Phi}=Y_{\Phi}^{\dagger} \\
\tilde{Y}_{\Phi}=\tilde{Y}_{\Phi}^{\dagger}
\end{array}, \quad C:\left\{\begin{array}{l}
Y_{\Delta_{R, L}}=Y_{\Delta_{L, R}}^{*} \\
Y_{\Phi}=Y_{\Phi}^{T} \\
\tilde{Y}_{\Phi}=\tilde{Y}_{\Phi}^{T}
\end{array} .\right.\right.
$$

In the mass eigenstate basis the flavor changing charged current Lagrangian is given by

$$
\mathcal{L}_{c c}=\frac{g}{\sqrt{2}}\left(\bar{\nu}_{L} V_{L}^{\dagger} W_{L} l_{L}+\bar{N}_{R} V_{R}^{\dagger} W_{R} l_{R}\right)+\text { h.c. },
$$

\footnotetext{
${ }^{1}$ See appendix B for a more detailed discussion on this issue.
} 
$V_{R}$ is the right-handed analogue of the PMNS mixing matrix $V_{L}$. In general it has three different mixing angles and six arbitrary complex phases and we parametrize it as

$$
V_{R}=K_{e} \hat{V}_{R} K_{N}
$$

with $K_{e} \equiv \operatorname{diag}\left(e^{i \phi_{e}}, e^{i \phi_{\mu}}, e^{i \phi_{\tau}}\right), K_{N} \equiv \operatorname{diag}\left(1, e^{i \phi_{2}}, e^{i \phi_{3}}\right)$. The matrix $\hat{V}_{R}$ has three mixing angles and the dirac phase $\delta$. We choose for $\hat{V}_{R}$ the standard form for the CKM matrix shown in the PDG [33].

The interaction terms of charged leptons with the doubly-charged scalars are

$$
\begin{aligned}
\mathcal{L}_{\Delta} & =\frac{1}{2} l_{R}^{T} C Y_{\Delta_{R}}^{\prime} \delta_{R}^{++} l_{R}+\frac{1}{2} l_{L}^{T} C Y_{\Delta_{L}}^{\prime} \delta_{L}^{++} l_{L}+\text { h.c. }, \\
Y_{\Delta_{R}}^{\prime} & =\frac{g}{M_{W_{R}}} V_{R}^{*} M_{N} V_{R}^{\dagger} .
\end{aligned}
$$

If charge conjugation is the discrete LR symmetry, the charged lepton masses are symmetric and in this case the Yukawa couplings in (3.6) satisfy (for reviews on this topic see references [23-25])

$$
Y_{\Delta_{L}}^{\prime}=\left(Y_{\Delta_{R}}^{\prime}\right)^{*}
$$

For parity and in the more interesting phenomenological situations, the charged lepton masses matrices are almost hermitian [34]. In [35] it was realized that it implies the near equality between the Yukawa couplings shown in eq. (3.6) i.e.

$$
Y_{\Delta_{L}}^{\prime}=Y_{\Delta_{R}}^{\prime}+\mathcal{O}(\tan 2 \beta \sin \alpha)
$$

The vacuum expectation values of the neutral fields belonging to $\Phi$ are such that $\left\langle\phi_{1}^{0}\right\rangle=v_{1}$ and $\left\langle\phi_{2}^{0}\right\rangle=v_{2} e^{i \alpha}$, where $\beta$ is the ratio $v_{2} / v_{1}$ and $\alpha$ is the spontaneous phase. In [36, 37] it is shown that $\tan 2 \beta \sin \alpha \lesssim 2 m_{b} / m_{t}$ ( $m_{b}$ and $m_{t}$ are the bottom and top quark masses respectively), so that the Yukawa coupling of the doubly charged scalar are nearly equal [35].

It is a remarkable feature of the minimal LR theory, that the TeV energy scale accessible at the LHC through the Keung-Senjanović (KS) process [38] -and its associated LNV and LFV, predicts the rate for the neutrino-less double beta decay and low energy LFV. This deep connection and the related phenomenology are illustrated in [39, 40]. All these processes depend in a crucial way on the elements of the leptonic right-handed mixing matrix $V_{R}$, for which all its mixing angles, the Dirac phase and two Majorana phases can be determined at the LHC [35]. Useful information can also be obtained from EDM of the neutron and such [41-45]. This is deeply connected to the study of the strong CP parameter, which in the mLRSM turns out to be calculable [46-48].

Recently the CMS collaboration [49] has reported an excess in the ee-channel for this process at $2.8 \sigma$, but they claimed that this excess cannot be accommodated in the minimal version of the theory -assuming diagonal mixing in the right-handed leptonic sector and degenerate masses for the heavy neutrinos. Several works have been proposed [50$53]$ in order to explain this excess and the conclusion was that it would need a higher Left-Right symmetry breaking scale, or a more general mixing scenario with pseudo-Dirac heavy neutrinos. 


\section{Computation of a triple vector correlation in the $\mu \rightarrow e \gamma$ decay}

$T$-odd asymmetries in the $\mu \rightarrow e \gamma$ were considered in the past. In $[8,9]$, it was shown that by studying the polarization of electron and the photon coming from the muon decay it is possible to extract the $\mathrm{CP}$-violating phases from the experiment. The conclusion was that in order to extract the CP-violating phases both electron and photon polarizations must be measured. In this paper instead, we present an alternative way of extracting the CPviolating phases of the effective Hamiltonian in the $\mu \rightarrow e \gamma$ decay. This is complementary to the work presented in $[8,9]$. The novelty is that no measurements of the final photon polarizations are needed. We consider the T-violating triple vector product

$$
\hat{s}_{\mu^{+}} \cdot\left(\hat{p}_{e^{+}} \times \hat{s}_{e^{+}}\right)=\cos \Phi \sin \theta_{s}
$$

where $\theta_{s}$ is the angle between the polarization's direction $\left(\hat{s}_{e^{+}}\right)$of the positron and its momentum's direction $\hat{p}_{e^{+}}, \Phi$ is the angle formed between $\hat{s}_{\mu^{+}}$and the direction defined by $\vec{p}_{e^{+}} \times \vec{s}_{e^{+}}$and $\Psi$ is the azimuthal angle. In figure 1 the reference frame and setup are shown. Notice that this quantity changes sign under parity and naive time-reversal transformation $\hat{T}$ defined by $t \rightarrow-t$. For processes whose interactions are characterized by a small coupling, it can be shown at first order that the connected part of the S-matrix is hermitian [10] and therefore the violation of the $\hat{T}$ symmetry amounts the violation of the time-reversal symmetry.

We define the triple vector correlation as

$$
\begin{aligned}
\left\langle\hat{s}_{\mu^{+}} \cdot\left(\hat{p}_{e^{+}} \times \hat{s}_{e^{+}}\right)\right\rangle_{\Phi} & \equiv \frac{N(\cos \Phi>0)-N(\cos \Phi<0)}{N_{\text {total }}} \\
& =\frac{\int_{0}^{\pi} d \Phi d \Gamma / d \Phi \cdot \operatorname{sgn}\left(\hat{s}_{\mu^{+}} \cdot\left(\hat{p}_{e} \times \hat{s}_{e^{+}}\right)\right)}{\Gamma_{\text {total }}},
\end{aligned}
$$

where $\Gamma_{\text {total }}$ and $N_{\text {total }}$ are the total decay rate and the total number of events for the initially polarized muon respectively, $N(\cos \Phi>0)$ and $N(\cos \Phi<0)$ are the number of events satisfying $\cos \Phi>0$ and $\cos \Phi<0$ respectively.

The 4-momenta of the participating particles in the rest frame of the muon are given by

$$
\begin{aligned}
p_{\mu^{+}}^{\mu} & =\left(m_{\mu}, 0,0,0\right), \\
p_{e^{+}}^{\mu} & =\left(E_{e},\left|\vec{p}_{e^{+}}\right| \sin \theta_{s},\left|\vec{p}_{e^{+}}\right| \cos \theta_{s}, 0\right) \\
p_{\gamma}^{\mu} & =\left(E_{\gamma},-\left|\vec{p}_{e^{+}}\right| \sin \theta_{s},-\left|\vec{p}_{e^{+}}\right| \cos \theta_{s}, 0\right)
\end{aligned}
$$

where the mass of the positron has been neglected. The energy $E_{e^{+}}$of the positron and the energy $E_{\gamma}$ of the photon are given by

$$
E_{e^{+}} \cong E_{\gamma}=\left|\vec{p}_{e^{+}}\right|=\frac{m_{\mu}}{2} .
$$

From the effective Hamiltonian in eq. (2.1) and eqs. (A.1), (A.4) and (A.5) in appendix A, a straightforward computation gives the following value for the correlation

$$
\left\langle\hat{s}_{\mu^{+}} \cdot\left(\hat{p}_{e^{+}} \times \hat{s}_{e^{+}}\right)\right\rangle_{\Phi}=\sin \theta_{s} \frac{\Im m\left(A_{L} A_{R}^{*}\right)}{\left|A_{L}\right|^{2}+\left|A_{R}\right|^{2}} .
$$




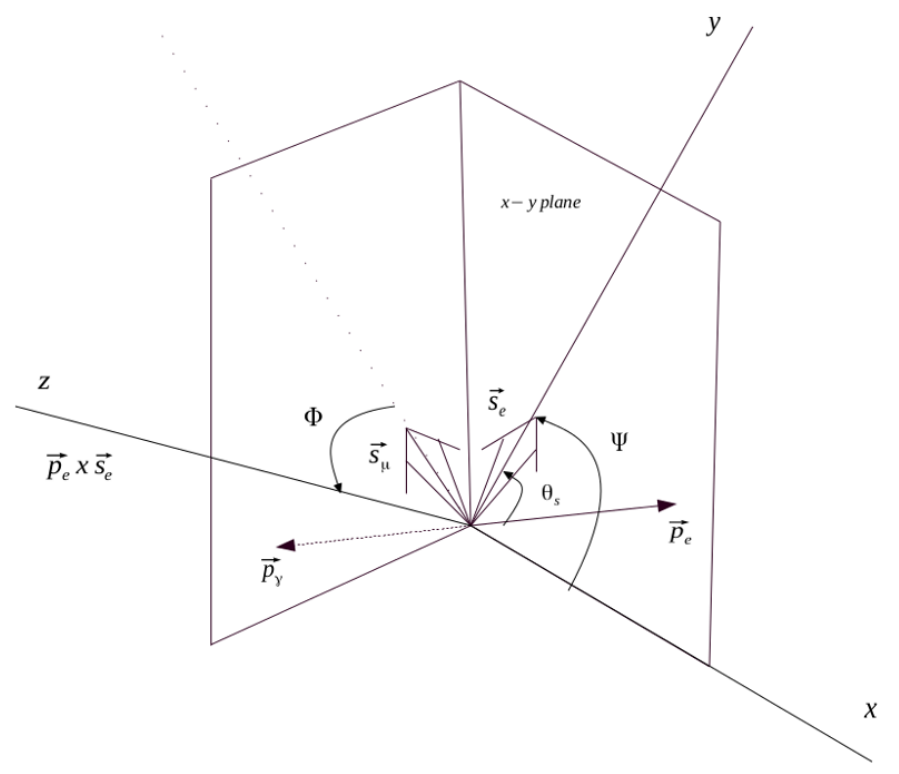

Figure 1. Reference frame and the setup for the $\mu \rightarrow e \gamma$ decay.

The main advantage of this quantity is that no measurements of the photon polarizations are needed.

In summary we find that given a source of polarized anti-muons, by measuring the 3momentum $\vec{p}_{e^{+}}$of the outgoing positron and its polarization $\vec{s}_{e^{+}}$, the asymmetry shown in eq. (4.7) is sensitive to the CP-violating phases of the effective Hamiltonian shown in (2.1). In [54-58] it is shown that measurements of the polarization of electrons coming from the muon decay are feasible. We assume a $100 \%$ polarized muon flux so that our results must be trivially rescaled by the actual polarization of the initial muons.

\section{Computation of a triple vector correlation in the $\mu \rightarrow e$ conversion process}

Following the same lines of the last section, we define an asymmetry given by comparing the number of events with $\vec{s}_{\mu} \cdot\left(\vec{p}_{e} \times \vec{s}_{e}\right)>0$ with the ones satisfying $\vec{s}_{\mu} \cdot\left(\vec{p}_{e} \times \vec{s}_{e}\right)<0$ in the $\mu \rightarrow e$ conversion process and we define it as

$$
\begin{aligned}
\left\langle\hat{s}_{\mu} \cdot\left(\hat{p}_{e} \times \hat{s}_{e}\right)\right\rangle_{\Phi} & \equiv \frac{N(\cos \Phi>0)-N(\cos \Phi<0)}{N_{\text {total }}} \\
& =\frac{\omega_{\text {conv }}(\cos \Phi>0)-\omega_{\text {conv }}(\cos \Phi<0)}{\omega_{\text {conv }}}
\end{aligned}
$$

where $\omega_{\text {conv }}$ is the total conversion rate and as previously, $\Phi$ is the angle between the plane formed by the vectors $\hat{p}_{e}$ and $\hat{s}_{e}$ and the polarization of the muon $\hat{s}_{\mu}$. We used the same coordinate system shown in figure 1 but clearly there is no photon coming from the muon decay. 
A direct computation of the asymmetry shown in eq. (5.1) gives ${ }^{2}$

$$
\left\langle\hat{s}_{\mu} \cdot\left(\hat{p}_{e} \times \hat{s}_{e}\right)\right\rangle_{\Phi}=\frac{1}{2} \sin \theta_{s} \frac{\Im m\left(C_{L} C_{R}^{*}\right)}{\left|C_{L}\right|^{2}+\left|C_{R}\right|^{2}}+\mathcal{O}(\alpha Z)+\mathcal{O}\left(\frac{m_{e}}{E_{e}}\right) .
$$

$m_{e}$ is the electron mass and

$$
\begin{aligned}
& C_{R} \equiv D A_{R}+S^{(p)}\left(\tilde{g}_{L S}^{(p)}+\tilde{g}_{L V}^{(p)}\right)+S^{(n)}\left(\tilde{g}_{L S}^{(n)}+\tilde{g}_{L V}^{(n)}\right), \\
& C_{L} \equiv D A_{L}+S^{(p)}\left(\tilde{g}_{R S}^{(p)}+\tilde{g}_{R V}^{(p)}\right)+S^{(n)}\left(\tilde{g}_{R S}^{(n)}+\tilde{g}_{R V}^{(n)}\right)
\end{aligned}
$$

where

$$
\begin{array}{ll}
\tilde{g}_{L S, R S}^{(p)} \equiv \sum_{q} G^{(q, p)} g_{L S, R S(q)}, & \tilde{g}_{L S, R S}^{(n)} \equiv \sum_{q} G^{(q, n)} g_{L S, R S(q)}, \\
\tilde{g}_{L V, R V}^{(p)} \equiv 2 g_{L V, R V(u)}+g_{L V, R V(d)}, & \tilde{g}_{L V, R V}^{(n)} \equiv g_{L V, R V(u)}+2 g_{L V, R V(d)} .
\end{array}
$$

$D, S^{(n, p)}$ are nuclear constants already calculated and tabulated in [15] for various elements. $G^{(q, p)}$ and $G^{(q, n)}$ are obtained from the scalar matrix element $[15,18]$

$$
\langle N|\bar{q} q| N\rangle=Z G^{(q, p)} \rho^{(p)}+(A-Z) G^{(q, n)} \rho^{(n)}
$$

$Z$ and $A$ are the atomic and mass number respectively, $\rho^{(n)}$ and $\rho^{(p)}$ are the neutron and proton densities inside the nucleus. The expression obtained is valid for non-relativistic muons and we droped terms of the order $\alpha Z$ and $m_{e} / E_{e}$. In practice equation (5.2) must be multiplied by the polarization of the initial muons, which is of the order of $15 \%$ in the conversion process [59].

\section{Triplet vector correlation in the minimal Left-Right theory}

As a concrete example of a theory beyond the SM that gives order one values for the T-odd triple vector correlation [11] we consider the minimal LR symmetric model. In what follows we analyze separately the contributions to the asymmetries (4.7) and (5.2) in the case of $P$ and $C$ as the LR symmetries. In [11] it is found that this contribution can be of order one, since there are new contributions coming from interactions of charged leptons with the singly-charged and doubly-charged scalar fields.

\section{1 $\mu \rightarrow e \gamma$ decay}

In this section and for the $\mu \rightarrow e \gamma$ decay, we study the contributions to the triple vector correlation for both Parity and Charge Conjugation as the LR symmetry.

Parity as the LR symmetry: in [60] the authors presented a complete study of the contributions to several LFV processes in the context of the minimal LR extension of the $\mathrm{SM}$ and it is found that the branching ratio for this process is of the form

$$
\operatorname{Br}(\mu \rightarrow e \gamma)=384 \pi^{2} e^{2}\left(\left|A_{L}\right|^{2}+\left|A_{R}\right|^{2}\right)
$$

\footnotetext{
${ }^{2}$ For more details see section B.2.
} 


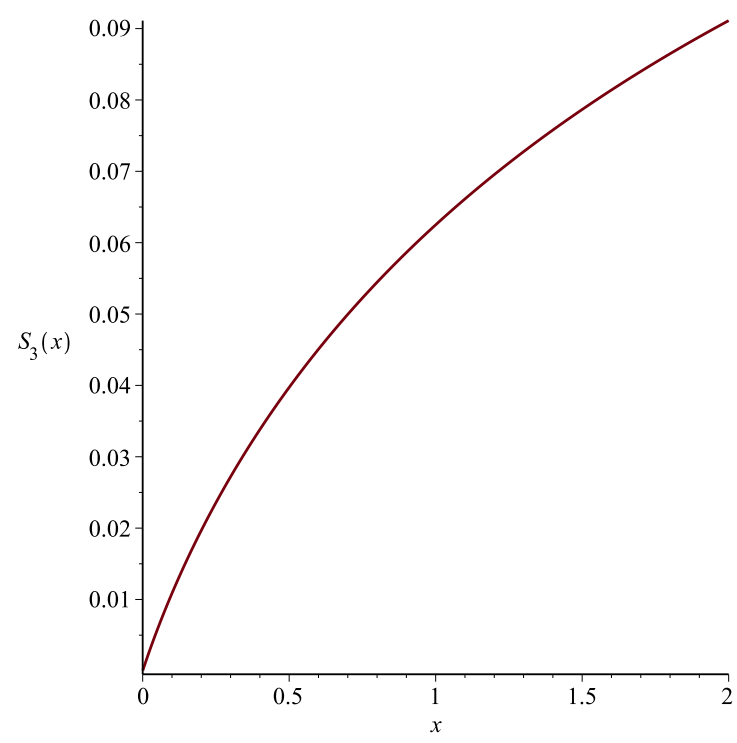

Figure 2. Plot of the loop function $S_{3}(x)$.

where

$$
\begin{aligned}
& A_{R}=\frac{1}{16 \pi^{2}} \sum_{n}\left(V_{R}^{\dagger}\right)_{e n}\left(V_{R}\right)_{n \mu}\left[\frac{M_{W}^{2}}{M_{W_{R}}^{2}} S_{3}\left(X_{n}\right)-\frac{X_{n}}{3} \frac{M_{W}^{2}}{M_{\delta_{R}^{++}}^{2}}\right], \\
& A_{L}=\frac{1}{16 \pi^{2}} \sum_{n}\left(V_{R}^{\dagger}\right)_{e n}\left(V_{R}\right)_{n \mu} X_{n}\left[-\frac{1}{3} \frac{M_{W}^{2}}{M_{\delta_{L}^{++}}^{2}}-\frac{1}{24} \frac{M_{W}^{2}}{M_{H_{1}^{+}}^{2}}\right]+\mathcal{O}(\tan 2 \beta \sin \alpha), \\
& X_{n}=\left(\frac{M_{N}}{M_{W_{R}}}\right)^{2}, \quad S_{3}(x)=-\frac{x}{8} \frac{1+2 x}{(1-x)^{2}}+\frac{3 x^{2}}{4(1-x)^{2}}\left[\frac{x}{(1-x)^{2}}(1-x+\log x)+1\right] .
\end{aligned}
$$

$M_{N_{n}}$ are the heavy neutrino masses where $n=1,2,3 . M_{W}$ is the $\mathrm{W}$ boson mass, $M_{W_{R}}$ is the $W_{R}$ boson mass, $M_{H_{1}^{+}}$is the mass of the heavy scalar $H_{1}^{+}$and $M_{\delta_{(L, R)}^{++}}$are the masses for the left and right doubly charged scalars respectively and finally we use $M_{\nu}$ to denote the light neutrino masses.

Notice that the loop function $S_{3}$ is always small as far as $M_{N}$ is not much bigger than $M_{W_{R}}$, so that the term with the loop function can neglected for a wide range of the heavy neutrino masses (see figure 2) and therefore the correlation defined in (4.7) is suppressed. Finally we neglect the contribution of the charged Higgs $H_{1}^{+}$since its mass cannot be lower than (15-20) $\mathrm{TeV}[36,61]$. This poses no problem for the theory, since its mass emerges at the large scale of symmetry breaking $[26,62]$. The gauge boson and doubly-charged scalar masses can be obtained at the LHC through the so called KS process and the decays of the doubly charged scalars [38] in addition with all the mixing angles and the Dirac phase in $V_{R}$ [35]. This is an example of the complementary role played by the high and low energy experiments in the establishment of the LR theory [39, 63-68].

For the sake of illustration, imagine that type II see-saw is the dominant source of neutrino masses i.e. $\frac{M_{N}}{\left\langle\Delta_{R}\right\rangle}=\frac{M_{\nu}}{\left\langle\Delta_{L}\right\rangle}$ and $V_{L}=V_{R}$. In this case it is possible to show that the 

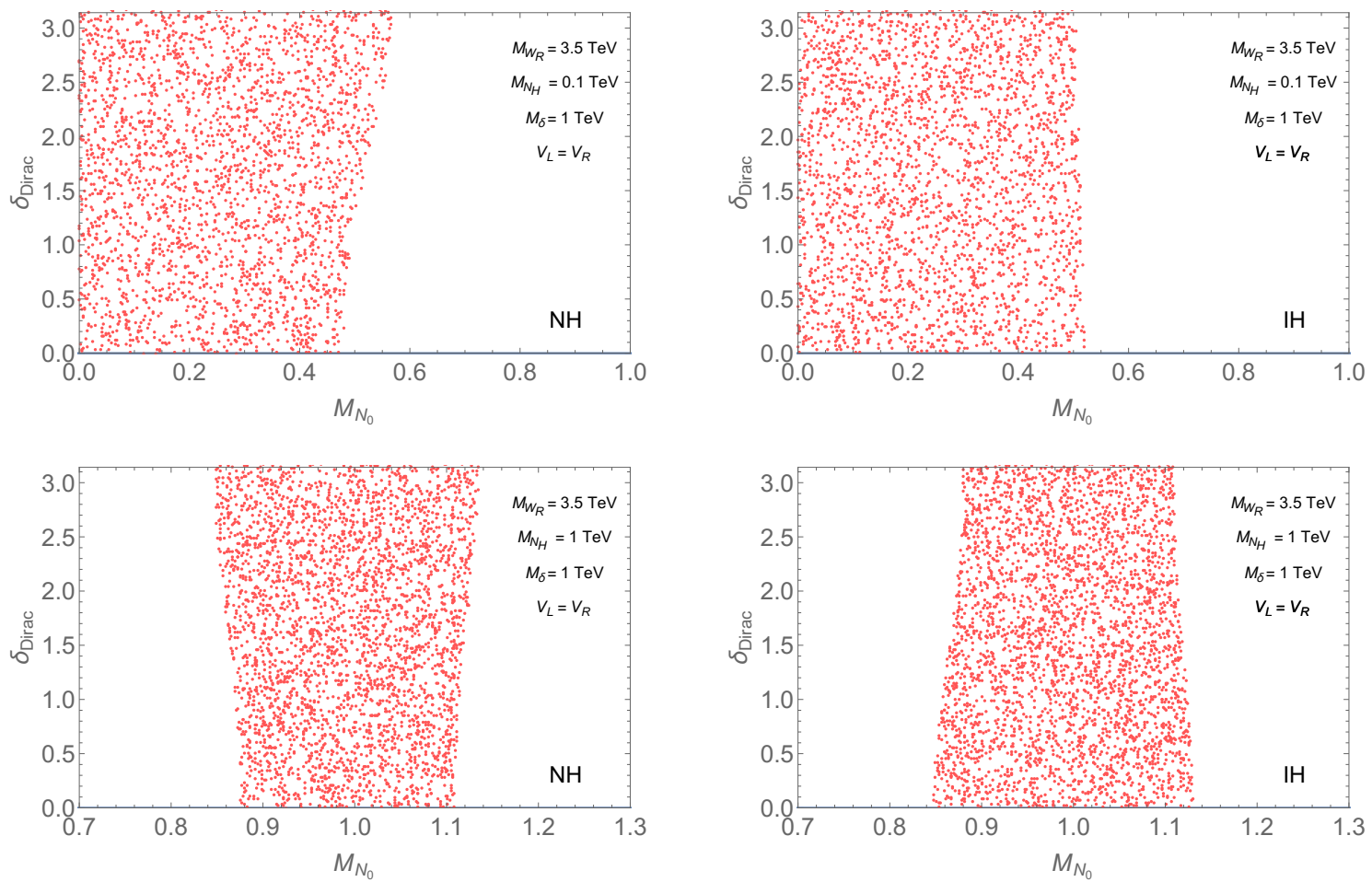

Figure 3. Plot obtained by considering the MEG bound shown in eq. (1.1). (Right) Normal hierarchy case (NH). (Left) Inverse hierarchy case (IH). The colored region is the allowed one. (Top) Mass of the heaviest right-handed neutrino $M_{N_{H}}=0.1 \mathrm{TeV}$. (Bottom) Mass of the heaviest right-handed neutrino $M_{N_{H}}=1 \mathrm{TeV}$.

heavy neutrino masses satisfy the relation [39]

$$
\frac{M_{N_{2}}^{2}-M_{N_{1}}^{2}}{M_{N_{3}}^{2}-M_{N_{1}}^{2}}=\frac{M_{\nu_{2}}^{2}-M_{\nu_{1}}^{2}}{M_{\nu_{3}}^{2}-M_{\nu_{1}}^{2}} \simeq \pm 0.03,
$$

where the \pm corresponds to normal $(\mathrm{NH})$ and inverted $(\mathrm{IH})$ neutrino mass hierarchy respectively. In what follows we denote $M_{N_{0}}$ the lightest right-handed neutrino mass, $M_{N_{H}}$ the heaviest right-handed neutrino mass and $\delta$ is the Dirac phase present in $\hat{V}_{R}$. In figure 3 and for the two representative values of $M_{N_{H}}=0.1 \mathrm{TeV}$ and $M_{N_{H}}=1 \mathrm{TeV}$ we show the allowed region obtained from the MEG bound in the $\left\{M_{N_{0}}, \delta_{\text {Dirac }}\right\}$ plane, for both normal and inverted neutrino mass spectrum. The region between these values gives rise to the exciting LNV signals at the LHC trough the KS process. We assume $M_{W_{R}}=3.5 \mathrm{TeV}$ and common masses for the doubly charged scalars $M_{\delta_{L}^{++}}=M_{\delta_{R}^{++}}=M_{\delta}=1 \mathrm{TeV}$. The reader may ask about the very different behavior obtained for the two values of the heaviest neutrino mass chosen, and the point is that this can be readily understood by noticing that the amplitude is approximately proportional to $\left|\Delta M_{13}^{2}\right|=\left|M_{N_{H}}^{2}-M_{N_{0}}^{2}\right|$, so that a bound is obtained for $\left|\Delta M_{13}^{2}\right|$ rather on the lightest neutrino mass itself.

In figure 4 (top) we plot the absolute value for the triple vector correlation given in (4.7) in the $\left(M_{N_{0}}, \delta\right)$-plane, where one may see that the values of the correlation (4.7) goes from $10^{-6}$ to $10^{-5}$ in the allowed region. 

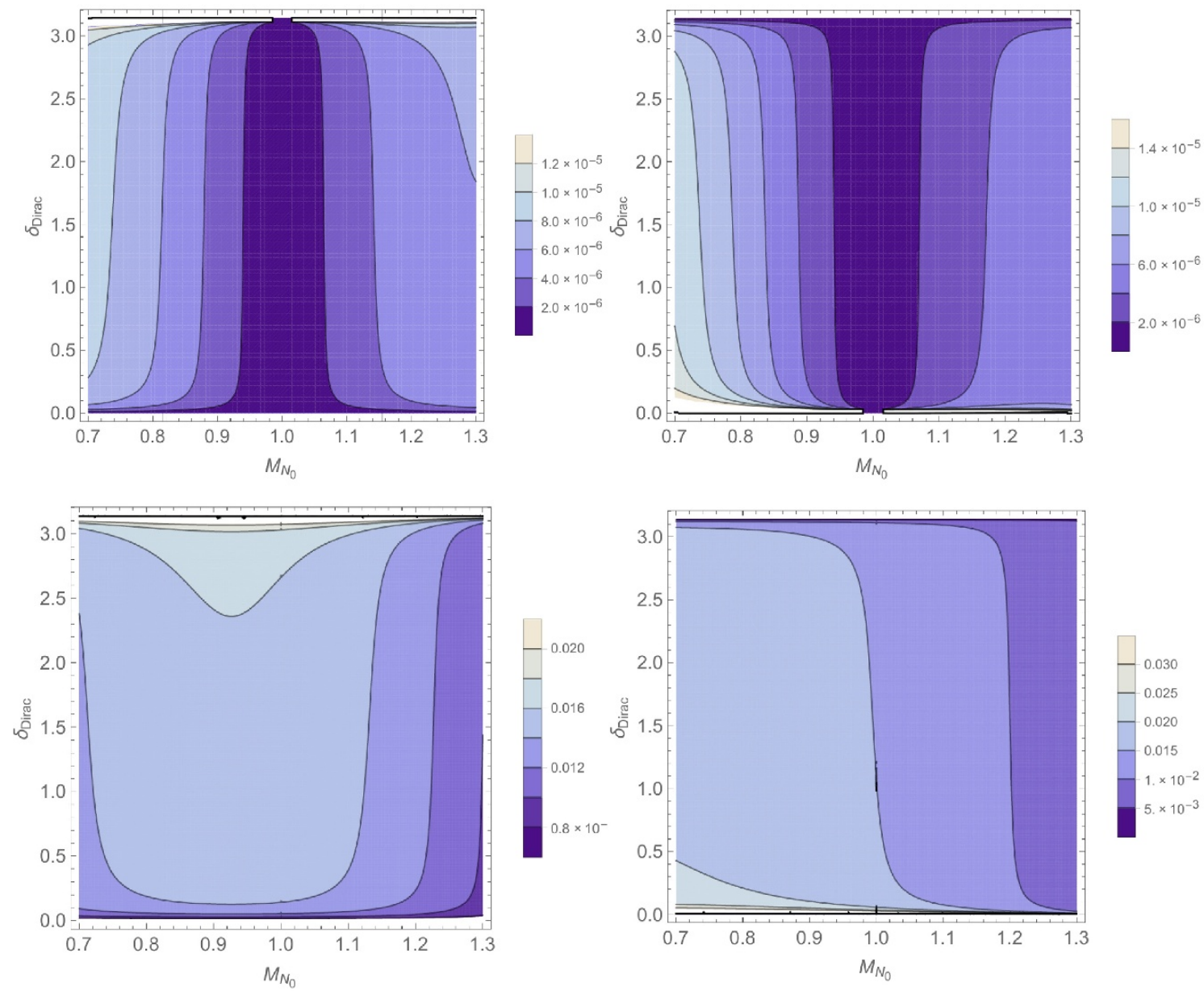

Figure 4. (Top) Contour plots illustrating the absolute value of the asymmetry defined in (4.7) as a function of the lightest neutrino mass $M_{N_{0}}$ and the Dirac phase $\delta$ for $P$ as the LR symmetry. (Bottom) Contour plots illustrating the value of the asymmetry defined in (4.7) as a function of the lightest neutrino mass $M_{N_{0}}$ and the Dirac phase $\delta$ (assuming $\phi_{\mu}-\phi_{e}=0$ ) for $C$ as the LR symmetry. (Left) Normal hierarchy for neutrino masses. (Right) Inverse hierarchy for neutrino masses. We take the gauge boson mass $M_{W_{R}}=3.5 \mathrm{TeV}$, the heaviest right-handed neutrino mass $M_{N_{H}}=1 \mathrm{TeV}$ and common masses for the doubly charged scalars of $M_{\delta}=1 \mathrm{TeV}$. The mixing angles are $\theta_{12} \simeq 33.6^{\circ}, \theta_{23} \simeq 41.9^{\circ}, \theta_{13} \simeq 8.7^{\circ}$.

One would be tempted to conclude that the triple vector correlation may be bigger for general values of neutrino masses and mixings. However from eqs. (3.9), the contribution to the triple vector correlation shown in (4.7) is bounded to be less $10^{-2}$ since $\tan 2 \beta \sin \alpha<$ $10^{-2}$ from the quark masses $[36,37,48]$. The point is that for charged leptons masses $\left(M_{l}\right)$ bigger or equal than the Dirac mass of neutrinos $\left(M_{D}\right)$, the mass matrix of the charged leptons is nearly hermitian leading therefore to nearly equal leptonic left and right mixing matrices. This is in complete analogy to the situation in the quark sector studied in [36, 41]. Of course it is possible to assume that $M_{D}>M_{l}$, but we will not pursue this possibility since in this case the original see-saw mechanism would lose its meaning and one would have to invoke accidental cancellations in order to explain the smallness neutrino masses. 
Charge conjugation as the LR symmetry: from eq. (3.8) we have that

$$
\begin{aligned}
& A_{R}=\frac{1}{16 \pi^{2}} \sum_{n}\left(V_{R}^{\dagger}\right)_{e n}\left(V_{R}\right)_{n \mu}\left[\frac{M_{W}^{2}}{M_{W_{R}}^{2}} S_{3}\left(X_{n}\right)-\frac{X_{n}}{3} \frac{M_{W}^{2}}{M_{\delta_{R}^{++}}^{2}}\right], \\
& A_{L}=\frac{1}{16 \pi^{2}} \sum_{n}\left(V_{R}^{T}\right)_{e n}\left(V_{R}^{*}\right)_{n \mu} X_{n}\left[-\frac{1}{3} \frac{M_{W}^{2}}{M_{\delta_{L}^{++}}^{2}}-\frac{1}{24} \frac{M_{W}^{2}}{M_{H_{1}^{+}}^{2}}\right] .
\end{aligned}
$$

Notice that some of the external phases appearing in $V_{R}$ do not cancel in (4.7) and the triple vector correlation is proportional to $e^{2\left(\phi_{\mu}-\phi_{e}\right)}$, so that the triple vector correlation is not suppressed by the small $\theta_{13}$ mixing-angle. In figure 4 (bottom) we show the absolute value of the triple vector correlation in the $\left(M_{N_{0}}, \delta\right)$-plane. We take $\left(\phi_{\mu}-\phi_{e}\right)=0$ in both normal and inverted neutrino mass hierarchies. For $\left(\phi_{\mu}-\phi_{e}\right)=\pi / 4$ it will reach in maximum value of around 0.5 in almost all the parameter space

Finally from figure 4 (bottom) we conclude that $C$ as the LR symmetry gives larger contributions to the triple vector correlation and this because in the parity case, the triple vector correlation is suppressed due to the near equality between the Yukawa couplings.

The bottom line is that in the most interesting region of the parameter space, a value for the triple vector correlation bigger than $10^{-2}$ can only be the consequence of $C$ as the LR symmetry.

One may ask whether this value for the asymmetry of could be measured in forthcoming experiments. Suppose that $\mu \rightarrow e \gamma$ is found to be of the order of $10^{-14}$. In the best scenario due to the future experimental improvements on the sensitivity, it would become possible to observed at most $10^{4}$ events and out of these events one has to select the ones that have $\theta_{s} \neq 0$ or $\theta_{s} \neq \pi$. Moreover suppose that only the events satisfying $\pi / 6<\theta_{s}<\pi / 3$ may be identify in the experiment due to its intrinsic sensitivity. This would imply that we end up having $10^{4} \int_{\pi / 6}^{\pi / 3} \sin \theta_{s} d \theta_{s} \sim 10^{3}$ events in the most optimistic situation. Hence this naive argument allow us to conclude that in most optimistic scenario, an asymmetry of the order $10^{-3}$ or bigger would probably be seen in the next round of $\mu \rightarrow e \gamma$ decay experiments.

\section{2 $\mu \rightarrow e$ conversion process}

In this section we consider the triple vector correlation for the $\mu \rightarrow e$ conversion process in the context of the minimal LR symmetric extension of the SM where the relevant branching ratio is given by [60]

$$
\operatorname{Br}(\mu \rightarrow e)=\frac{2 G_{F}^{2} V^{(p) 2}}{\Gamma_{\text {capt }}}\left(\frac{\alpha^{2}}{16 \pi^{2}}\right)\left(\left|F_{L}^{(\gamma)}\right|^{2}+\left|F_{R}^{(\gamma)}\right|^{2}\right) .
$$

The values of the capture rate $\Gamma_{\text {capt }}$ are tabulated in [69] for several elements. In [60] it was shown that the contribution of the doubly-charged scalar may dominate due to a logarithmic enhancement and in this case the functions $F_{L}^{(\gamma)}$ and $F_{R}^{(\gamma)}$ may be written as

$$
F_{(L, R)}^{(\gamma)} \simeq 128 \pi^{2} A_{(L, R)} \log \left(m_{\mu}^{2} / M_{\delta_{(L, R)}^{++}}^{2}\right)
$$



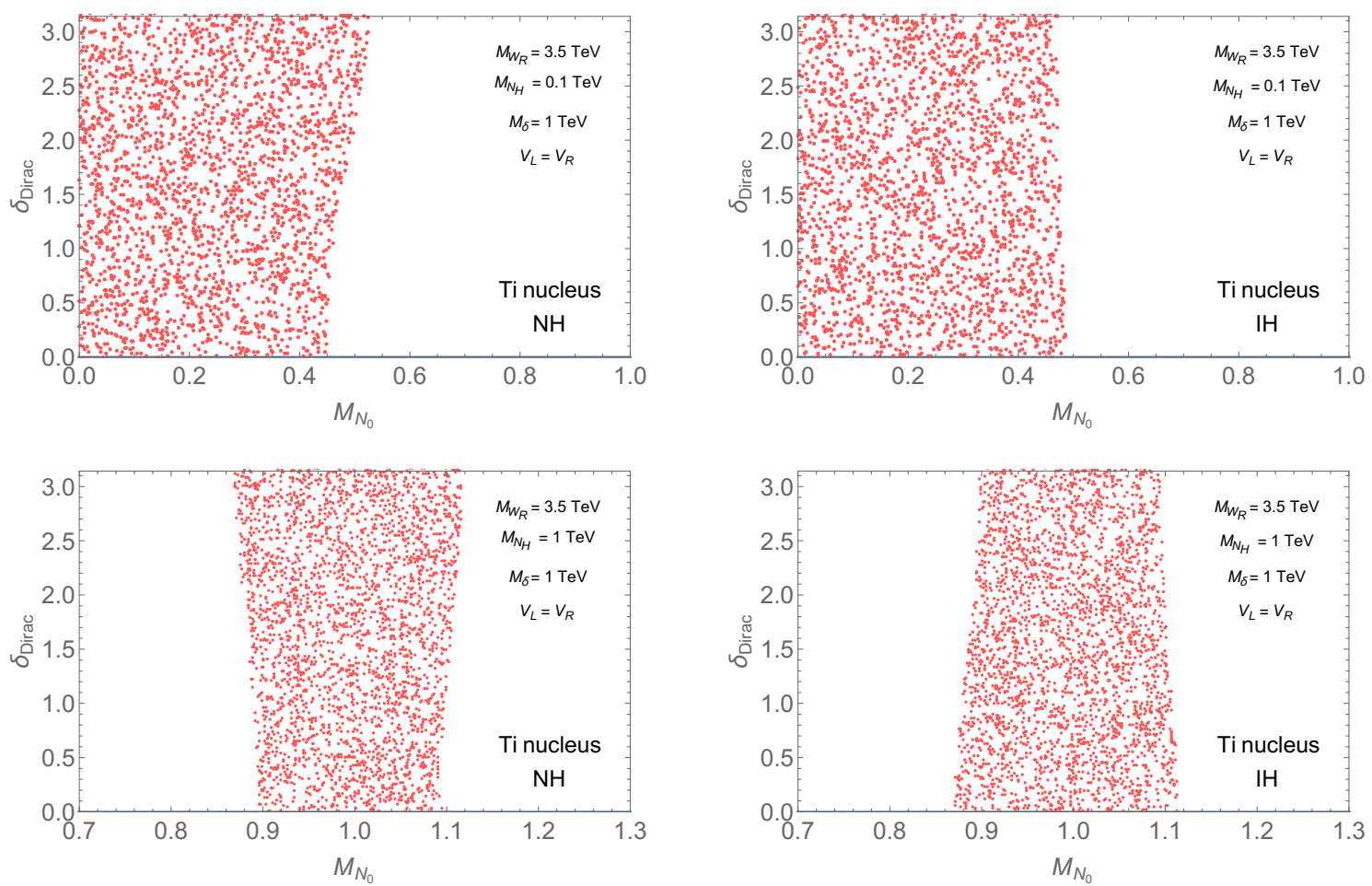

Figure 5. Plot obtained by considering the SINDRUM II bound for Titanium shown in eq. (1.2). (Right) Normal hierarchy case (NH). (Left) Inverse hierarchy case (IH). The colored region is the allowed one. (Top) Mass of the heaviest right-handed neutrino $M_{N_{H}}=0.1 \mathrm{TeV}$. (Bottom) Mass of the heaviest right-handed neutrino $M_{N_{H}}=1 \mathrm{TeV}$.

For completeness we show in figure 5 the allowed region obtained by considering the SINDRUM bound for Titanium shown in eq. (1.2) assuming the same values for the heavy neutrino masses of the last section. From eq. (6.9) and assuming that the dominant terms are the logarithmic enhance ones, the amplitude for the conversion process and the $\mu \rightarrow e \gamma$ decay are proportional. Therefore a similar qualitative behavior is obtained. We can see that the bound obtained is similar to the one of the $\mu \rightarrow e \gamma$ experiment and this is due to the fact that the logarithmic enhancement in eq. (6.9) compensate the $\alpha$ suppression in the conversion rate [60]. For Gold the bound one would obtained is similar since the ratio between the conversion rates for the two elements is around 0.83 . On the other hand, for the gold atom relativistic effects of the muon becomes relevant, so that the result shown in eq. (5.2) cannot be trusted in this case.

Finally the asymmetry defined in eq. (5.2) takes the form

$$
\left\langle\vec{s}_{\mu} \cdot\left(\vec{p}_{e} \times \vec{s}_{e}\right)\right\rangle_{\Phi}=\frac{\sin \theta_{s}}{2} \frac{\Im m\left(F_{L}^{(\gamma)} F_{R}^{*(\gamma)}\right)}{\left|F_{L}^{(\gamma)}\right|^{2}+\left|F_{R}^{(\gamma)}\right|^{2}}=\frac{\sin \theta_{s}}{2} \frac{\Im m\left(A_{L} A_{R}^{*}\right)}{\left|A_{L}\right|^{2}+\left|A_{R}\right|^{2}},
$$

where it can be seen that this asymmetry has the same flavor structure of the coefficients $A_{L}$ and $A_{R}$ defined previously for the $\mu \rightarrow e \gamma$ decay, therefore the same conclusion obtained in the $\mu \rightarrow e \gamma$ case holds for the $\mu \rightarrow e$ conversion process as well. 
Regarding the expected sensitivity for the conversion process the arguments we used in the $\mu \rightarrow e \gamma$ decay apply, but with the difference that the final sensitivity is rescaled by a factor of the order of $10^{-1}$ due to the depolarization -around $15 \%$ - of the muons in the conversion process [59].

\section{Conclusions}

We derived analytical expressions for a T-odd triple vector correlation in the $\mu \rightarrow e \gamma$ decay and the $\mu \rightarrow e$ conversion process and found simple results in terms of the CP-violating phases of the effective Hamiltonians. The expression obtained in the $\mu \rightarrow e$ conversion omits relativistic corrections for the muons, but is otherwise complete. For the $\mu \rightarrow e \gamma$ decay we conclude that in order to extract the $\mathrm{CP}$ violating phases of the theory from the experiment, no measurements of the photon polarizations are needed.

Then as an example of a theory that leads order one values for the triple vector correlation we consider the TeV scale, minimal Left-Right symmetric extension of the SM. Remarkably, due to the relation between left and right Yukawa couplings in (3.6) -see also eqs. (3.8) and (3.9) - this triple vector correlation can be used to discriminate between charge-conjugation or parity as the Left-Right symmetry. More precisely, for Dirac masses of neutrinos smaller or of the order of the charge lepton masses, a value for the triple vector correlation bigger than $10^{-2}$ can only be the consequence of charge-conjugation as the Left-Right symmetry.

\section{A Kinematics of the $\mu \rightarrow e \gamma$ process and the triple vector correlation}

In this appendix we give some tools that could be useful when computing the triple vector correlation shown in eq. (4.7) for the $\mu \rightarrow e \gamma$ decay.

For the anti-muon we use the spinor $v\left(p_{\mu^{+}}\right)$given by

$$
v\left(p_{\mu^{+}}\right)=\left(\begin{array}{cc}
\sqrt{p \cdot \sigma} & \xi \\
-\sqrt{p \cdot \bar{\sigma}} & \xi
\end{array}\right),
$$

where $\xi^{\dagger} \xi=1$ and $p_{\mu^{+}}$is given in eq. (4.3). As shown in figure 1 the polarization vector of the muon is given by:

$$
\vec{s}=|\vec{s}|(\sin \Phi \cos \Psi, \sin \Phi \sin \Psi, \cos \Phi)
$$

and it is straightforward to show that in this case

$$
\xi^{n}=\left(\begin{array}{c}
e^{-i \frac{\Psi}{2}} \cos \frac{\Phi}{2} \\
e^{i \frac{\Psi}{2}} \sin \frac{\Phi}{2}
\end{array}\right) .
$$

One may find the same result by requiring $\xi$ to be an eigenvector of $\vec{\sigma} \cdot \hat{n}$, where $\hat{n}$ is a unitary vector in the direction of $\vec{s}$. 
For the electron and for the reference frame shown in figure 1 we use

$$
v_{e^{+}}\left(p_{e^{+}}\right)=\sqrt{\frac{\left|\vec{p}_{e^{+}}\right|}{2}}\left(\begin{array}{c}
-2 e^{i \frac{\theta_{s}}{2}} \sin \frac{\theta_{s}}{2} \\
2 i e^{i \frac{\theta_{s}}{2}} \sin \frac{\theta_{s}}{2} \\
2 i e^{i \frac{\theta_{s}}{2}} \cos \frac{\theta_{s}}{2} \\
-2 e^{-i \frac{\theta_{s}}{2}} \cos \frac{\theta_{s}}{2}
\end{array}\right) .
$$

The photon has two possible polarizations along the direction of motion and in the particular frame we are considering in figure 1 its polarization vector is given by,

$$
\epsilon_{ \pm}^{\mu}\left(p_{\gamma}\right)=\frac{1}{\sqrt{2}}\left(\begin{array}{c}
0 \\
\pm i \cos \theta_{s} \\
\mp i \sin \theta_{s} \\
1
\end{array}\right)
$$

where we can explicitly see that when $\theta_{s}=0$, the photon can only have a polarization \pm 1 along the $\mathrm{y}$-axis and $p_{\gamma}$ and $p_{e^{+}}$are the 4-momentum of the outgoing photon and electron respectively - see eq. (4.4) and (4.5). Once the expressions for the spinors of the participating fermions and the polarization vector of the photon are known, it is easy straightforward to compute the triple vector asymmetry given in (4.7).

We found that the total decay rate is given by

$$
\Gamma_{\text {total }}=\frac{2}{\pi} G_{F}^{2} m_{\mu}^{5} e^{2}\left(\left|A_{L}\right|^{2}+\left|A_{R}\right|^{2}\right) .
$$

It would be interesting to compare the above equation with the result one gets when summing the decay rates for $\cos \Phi>0$ to that of $\cos \Phi<0$, namely

$$
\Gamma(\cos \Phi>0)+\Gamma(\cos \Phi<0)=\frac{2}{\pi} G_{F}^{2} m_{\mu}^{5} e^{2}\left(\cos ^{2} \frac{\theta_{s}}{2}\left|A_{L}\right|^{2}+\sin ^{2} \frac{\theta_{s}}{2}\left|A_{R}\right|^{2}\right) .
$$

On the other hand, by subtracting the total decay rates for $\cos \Phi>0$ to that of $\cos \Phi<0$ one gets:

$$
\Gamma(\cos \Phi>0)-\Gamma(\cos \Phi<0)=\frac{2}{\pi} G_{F}^{2} m_{\mu}^{5} e^{2} \sin \theta_{s} \Im m\left(A_{L} A_{R}^{*}\right)
$$

from which the asymmetry shown in (4.7) can be readily computed.

\section{B $\quad \mu \rightarrow e$ total conversion rate and the triple vector correlation}

\section{B.1 Total conversion rate}

In this appendix we briefly comment about the amplitude of the $\mu \rightarrow e$ conversion process and the Born's approximation we used.

In computing the $\mu \rightarrow e$ conversion process, one usually assumes the so called Born's approximation for the outgoing electrons. This approximation has two meanings: one is computing the conversion rate to a given order in some small coupling; and the other is the assumption that electrons coming from the conversion process are plane waves. The point is that we can do better and have a complete control of both approximations at the 
same time. More precisely for the relativistic one-electron atom and in the limit of big $r$ $\left(r \gg r_{0}\right.$, where $\left.V\left(r \geq r_{0}\right)=0\right)$, the solution of the Dirac's equation at first order in the perturbation $H_{\text {eff }}$ is of the form [16]

$$
\psi_{\text {as }}=-i \sqrt{\frac{\pi}{|\vec{p}|}} \frac{e^{i p r}}{r} \sum_{\kappa \mu} e^{i \delta_{\kappa}}\left\langle\psi_{\kappa}^{\mu}\left|H_{\mathrm{eff}}\right| \psi_{i}\right\rangle\left(\begin{array}{c}
\sqrt{E+1} \chi_{\kappa}^{\mu}(\hat{p}) \\
-\sqrt{E-1} \chi_{-\kappa}^{\mu}(\hat{p})
\end{array}\right)+\mathcal{O}\left(H_{\mathrm{eff}}^{2}\right),
$$

where $\psi_{i}$ is any stationary state of the Coulomb potential, $\psi_{\kappa}^{\mu}$ is one of the continuum energy solutions and $H_{\text {eff }}$ is the effective Hamiltonian for the $\mu \rightarrow e$ conversion process. Furthermore it can be shown that $\psi_{\text {as }}$ is an eigenfunction of $\vec{\alpha} \cdot \vec{p}+\beta$ with eigenvalue $E$ so that $\psi_{\text {as }}$ describes, indeed a plane wave [16]. In the high energy limit -neglecting the electron mass- the solution $\psi_{\text {as }}$ simplifies to

$$
\psi_{\text {as }}=-i \sqrt{\pi} \frac{e^{i p r}}{r} \sum_{\kappa \mu} e^{i \delta_{\kappa}}\left\langle\psi_{\kappa}^{\mu}\left|H_{\mathrm{eff}}\right| \psi_{i}\right\rangle\left(\begin{array}{c}
\chi_{\kappa}^{\mu}(\hat{p}) \\
-\chi_{-\kappa}^{\mu}(\hat{p})
\end{array}\right) .
$$

Finally if we are interested in computing the total conversion amplitude per unit flux (for a detector placed at fixed radius $r=R$ ) the total conversion rate is given by

$$
\omega_{\mathrm{conv}}=R^{2} \int d \Omega \psi_{\text {as }}^{\dagger} \psi_{\text {as }}=2 \pi\left(\frac{1}{2} \sum_{\kappa, \mu}\left|\left\langle\psi_{\kappa}^{\mu}\left|H_{\mathrm{eff}}\right| \psi_{i}\right\rangle\right|^{2}\right)=2 G_{F}^{2}\left(\left|C_{L}\right|^{2}+\left|C_{R}\right|^{2}\right)
$$

where the coefficients $C_{L}$ and $C_{R}$ are defined in section 5 and we may absorb the $\sqrt{2 \pi}$ factor into the normalization of the wave function $\psi_{\kappa}^{\mu}$ in order to agree with the conventions adopted in [15].

\section{B.2 Triple vector correlation in the conversion process}

In this appendix we give details of the calculation for the triplet correlation asymmetry in the $\mu \rightarrow e$ conversion process within the formalism developed in [16]. Since we are interested in describing particles with a given polarization, we are going to make use of the spin projection operators for Dirac spinors. Instead of using the covariant spin projection operator we make use of the following projection operator

$$
P_{\hat{n}_{0}}^{( \pm)}=\frac{1}{2}\left(1 \pm \mathcal{O} \cdot \hat{n}_{0}\right)
$$

where

$$
\mathcal{O} \equiv \beta \vec{\sigma}+(1-\beta)(\vec{\sigma} \cdot \hat{p}) \hat{p}
$$

and $\hat{n_{0}}$ is the direction of the spin polarization vector in the rest frame of the particle, $\hat{p}$ is the direction of its momentum and the \pm represent positive and negative polarization respectively. Furthermore it can be shown that the description of the spin with this operator is equivalent to the usual one given by the manifestly covariant spin operator. ${ }^{3}$ Notice that the non-relativistic limit of can be taken in a transparent way by replacing $\beta \rightarrow 1$.

\footnotetext{
${ }^{3}$ See [17] chapter III.
} 
For our present problem we assumed the muon to be non-relativistic and in the frame shown in figure 1 its polarization vector is of the form

$$
n_{\mu}=\left(0, \hat{n}_{0}\right)
$$

where

$$
\hat{n}_{0}=(\sin \Phi \cos \Psi, \sin \Phi \sin \Psi, \cos \Phi)
$$

by multiplying the wave function of the muon in the conversion process by $P_{\hat{n}_{0}}^{(+)}$one obtains the wave function of a non-relativistic muon with the given polarization. For the electron instead a full relativistic treatment is required since its energy is $E_{e}=m_{\mu}-\epsilon_{b}$, where $m_{\mu}$ is the muon mass and $\epsilon_{b}$ is the binding energy of the muon in the 1s state of the muonic atom. In this case the spin projection operator coming from the conversion process is given by

$$
P_{e}^{(+)}=\frac{1}{2}\left(1+\mathcal{O}^{e} \cdot \hat{n}_{0}^{e}\right)
$$

and

$$
\begin{aligned}
\mathcal{O}^{e} \cdot \hat{n}_{0}^{e} & =\beta \vec{\sigma} \cdot \hat{n}_{0}^{e}+(1-\beta)\left(\vec{\sigma} \cdot \hat{p}_{e}\right)\left(\hat{p}_{e} \cdot \hat{n}_{0}^{e}\right), \\
\hat{n}_{0}^{e} & =(0,1,0), \quad \hat{p}_{e}=\left(\sin \theta_{s}, \cos \theta_{s}, 0\right) .
\end{aligned}
$$

Finally the wave function describing the polarized outgoing electron -coming from the conversion of a polarized muon- is obtained by applying $P_{e}^{(+)}$to the solution (2.14) and a direct computation shows (for a detector placed at a fixed radius $\mathrm{R}$ ):

$$
\begin{aligned}
& \omega_{\text {conv }}(\cos \Phi>0)-\omega_{\text {conv }}(\cos \Phi<0)=R^{2} \int d \Omega \cdot \operatorname{sgn}\left(\hat{s}_{\mu} \cdot\left(\hat{p}_{e} \times \hat{s}_{e}\right)\right) \cdot \psi_{a s}^{\dagger} P_{e}^{(+)} \psi_{a s} \\
& =\frac{1}{2} G_{F}^{2} \sin \theta_{s} \Re e\left[e^{i\left(\delta_{-1}-\delta_{+1}\right)}\left(C_{R}-C_{L}\right)\left(\left(C_{R}^{*}+C_{L}^{*}\right)\right)\right]=G_{F}^{2} \sin \theta_{s} \Im m\left(C_{L} C_{R}^{*}\right)+\mathcal{O}(\alpha Z) \\
& \quad+\mathcal{O}\left(\frac{m_{e}}{E_{e}}\right)
\end{aligned}
$$

since in the high energy limit the Coulomb phases satisfy

$$
\delta_{-1}-\delta_{+1}=\frac{\pi}{2}+\mathcal{O}\left(\frac{\alpha Z}{E_{e}}\right) .
$$

The Coulomb phases $\delta_{ \pm 1}$ are defined in eq. (2.15) and $d \Omega$ is given by $d \Omega=d \Psi d \Phi \sin \Phi$.

\section{Acknowledgments}

I would like to thank G. Senjanović for suggesting me the subject, his continuous interest, advice and encouragement during the completion of this work. Thanks are due to G. Senjanović and S. Bertolini for enlightening and useful discussions and to G. Senjanović, S. Bertolini and A. Melfo for a careful reading of the manuscript. 
Open Access. This article is distributed under the terms of the Creative Commons Attribution License (CC-BY 4.0), which permits any use, distribution and reproduction in any medium, provided the original author(s) and source are credited.

\section{References}

[1] MEG collaboration, J. Adam et al., New constraint on the existence of the $\mu^{+} \rightarrow e^{+} \gamma$ decay, Phys. Rev. Lett. 110 (2013) 201801 [arXiv:1303.0754] [INSPIRE].

[2] SINDRUM II collaboration, W.H. Bertl et al., A search for muon to electron conversion in muonic gold, Eur. Phys. J. C 47 (2006) 337 [INSPIRE].

[3] P. Wintz Results of the SINDRUM-II experiment, in proceedings of the First International Symposium on Lepton and Baryon Number Violation, H.V. Klapdor-Kleingrothaus and I.V. Krivosherina eds, Inst. Phys. Publ., London U.K. (1998), pg. 534.

[4] A. de Gouvêa and N. Saoulidou, Fermilab's intensity frontier, Ann. Rev. Nucl. Part. Sci. 60 (2010) 513 [INSPIRE].

[5] J. Miller et al., Intense Muon Beams for Experiments at Project X, Conf. Proc. C 110328 (2011) 1951.

[6] H. Witte et al., Status of the PRISM FFAG Design for the Next Generation Muon-to-Electron Conversion Experiment, Conf. Proc. C 1205201 (2012) 79.

[7] S. Mihara, J.P. Miller, P. Paradisi and G. Piredda, Charged Lepton Flavor-Violation Experiments, Ann. Rev. Nucl. Part. Sci. 63 (2013) 531 [InSPIRE].

[8] Y. Farzan, Tracing CP-violation in lepton flavor violating muon decays, JHEP 07 (2007) 054 [hep-ph/0701106] [INSPIRE].

[9] S.Y. Ayazi and Y. Farzan, A Window on the CP-violating Phases of MSSM from Lepton Flavor Violating Processes, JHEP 01 (2009) 022 [arXiv:0810.4233] [INSPIRE].

[10] L. Wolfenstein, Final state interactions and CP-violation in weak decays, Phys. Rev. D 43 (1991) 151 [INSPIRE].

[11] B. Bajc, M. Nemevšek and G. Senjanović, Probing leptonic CP phases in LFV processes, Phys. Lett. B 684 (2010) 231 [arXiv:0911.1323] [INSPIRE].

[12] S. Weinberg and G. Feinberg, Electromagnetic Transitions Between mu Meson and Electron, Phys. Rev. Lett. 3 (1959) 111 [inSPIRE].

[13] W.J. Marciano and A.I. Sanda, The Reaction $m u^{-}$Nucleus $\rightarrow e^{-}$Nucleus in Gauge Theories, Phys. Rev. Lett. 38 (1977) 1512 [INSPIRE].

[14] O.U. Shanker, Z Dependence of Coherent $\mu$ Conversion Rate in Anomalous Neutrinoless Muon Capture, Phys. Rev. D 20 (1979) 1608 [inSPIRE].

[15] R. Kitano, M. Koike and Y. Okada, Detailed calculation of lepton flavor violating muon electron conversion rate for various nuclei, Phys. Rev. D 66 (2002) 096002 [Erratum ibid. D 76 (2007) 059902] [hep-ph/0203110] [INSPIRE].

[16] M.E Rose, L.C. Biedenharn and G.B. Arfken, Internal Conversion Angular Correations, Phys. Rev. 85 (1952) 5.

[17] M.E. Rose, Relativistic Electron Theory, John Wiley, New York, U.S.A. (1961), sections I and $\mathrm{V}$. 
[18] T.S. Kosmas, S. Kovalenko and I. Schmidt, Nuclear $\mu^{-}-e^{-}$conversion in strange quark sea, Phys. Lett. B 511 (2001) 203 [hep-ph/0102101] [INSPIRE].

[19] J.C. Pati and A. Salam, Lepton Number as the Fourth Color, Phys. Rev. D 10 (1974) 275 [Erratum ibid. D 11 (1975) 703] [INSPIRE].

[20] R.N. Mohapatra and J.C. Pati, Left-Right Gauge Symmetry and an Isoconjugate Model of CP-violation, Phys. Rev. D 11 (1975) 566 [InSPIRE].

[21] R.N. Mohapatra and J.C. Pati, A Natural Left-Right Symmetry, Phys. Rev. D 11 (1975) 2558 [INSPIRE].

[22] G. Senjanović and R.N. Mohapatra, Exact Left-Right Symmetry and Spontaneous Violation of Parity, Phys. Rev. D 12 (1975) 1502 [inSPIRE].

[23] G. Senjanović, Seesaw at LHC through Left-Right Symmetry, Int. J. Mod. Phys. A 26 (2011) 1469 [arXiv: 1012.4104] [INSPIRE].

[24] G. Senjanović, Neutrino mass: From LHC to grand unification, Riv. Nuovo Cim. 34 (2011) 1 [INSPIRE].

[25] V. Tello, Connections Between the High and Low Energy Violation of Lepton and Flavor Numbers in the Minimal Left-Right Symmetric Model, Ph.D. Thesis, SISSA, Italy (2012).

[26] G. Senjanović, Spontaneous Breakdown of Parity in a Class of Gauge Theories, Nucl. Phys. B 153 (1979) 334 [INSPIRE].

[27] P. Minkowski, $\mu \rightarrow$ e $\gamma$ at a Rate of One Out of $10^{9}$ Muon Decays?, Phys. Lett. B 67 (1977) 421 [INSPIRE].

[28] R.N. Mohapatra and G. Senjanović, Neutrino Mass and Spontaneous Parity Violation, Phys. Rev. Lett. 44 (1980) 912 [INSPIRE].

[29] R.N. Mohapatra and G. Senjanović, Neutrino Masses and Mixings in Gauge Models with Spontaneous Parity Violation, Phys. Rev. D 23 (1981) 165 [INSPIRE].

[30] S. Glashow, Quarks and leptons, Cargése 1979, M. Lévy ed., Plenum, NY, U.S.A. (1980).

[31] M. Gell-Mann,P. Ramond and R. Slansky, Complex Spinors and Unified Theories, in proceedings of the Supergravity Stony Brook workshop, New York, U.S.A. 1979), P. Van Niewenhuizen and D. Freeman eds., North Holland, Amsterdam, (1980).

[32] T. Yanagida, Horizontal Symmetry And Masses Of Neutrinos, in Workshop on unifed theories and baryon number in the universe, A. Sawada and A. Sugamoto eds., KEK, Tsukuba, Japan 1979.

[33] Particle Data Group collaboration, K.A. Olive et al., Review of Particle Physics, Chin. Phys. C 38 (2014) 090001.

[34] V. Tello, G. Senjanović and M. Nemevšek, work to appear.

[35] J.C. Vasquez, Right-handed lepton mixings at the LHC, arXiv:1411.5824 [INSPIRE].

[36] A. Maiezza, M. Nemevšek, F. Nesti and G. Senjanović, Left-Right Symmetry at LHC, Phys. Rev. D 82 (2010) 055022 [arXiv: 1005.5160] [INSPIRE].

[37] G. Senjanović and V. Tello, Right Handed Quark Mixing in Left-Right Symmetric Theory, Phys. Rev. Lett. 114 (2015) 071801 [arXiv: 1408.3835] [INSPIRE].

[38] W.-Y. Keung and G. Senjanović, Majorana Neutrinos and the Production of the Right-handed Charged Gauge Boson, Phys. Rev. Lett. 50 (1983) 1427 [INSPIRE]. 
[39] V. Tello, M. Nemevšek, F. Nesti, G. Senjanović and F. Vissani, Left-Right Symmetry: from LHC to Neutrinoless Double Beta Decay, Phys. Rev. Lett. 106 (2011) 151801 [arXiv:1011.3522] [INSPIRE].

[40] M. Nemevšek, F. Nesti, G. Senjanović and V. Tello, Neutrinoless Double Beta Decay: Low Left-Right Symmetry Scale?, arXiv:1112.3061 [INSPIRE].

[41] Y. Zhang, H. An, X. Ji and R.N. Mohapatra, General CP-violation in Minimal Left-Right Symmetric Model and Constraints on the Right-Handed Scale, Nucl. Phys. B 802 (2008) 247 [arXiv: 0712.4218] [INSPIRE].

[42] H. An, X. Ji and F. Xu, P-odd and CP-odd Four-Quark Contributions to Neutron EDM, JHEP 02 (2010) 043 [arXiv: 0908.2420] [INSPIRE].

[43] F. Xu, H. An and X. Ji, Neutron Electric Dipole Moment Constraint on Scale of Minimal Left-Right Symmetric Model, JHEP 03 (2010) 088 [arXiv:0910.2265] [INSPIRE].

[44] C.-Y. Seng, J. de Vries, E. Mereghetti, H.H. Patel and M. Ramsey-Musolf, Nucleon electric dipole moments and the isovector parity- and time-reversal-odd pion-nucleon coupling, Phys. Lett. B 736 (2014) 147 [arXiv:1401.5366] [INSPIRE].

[45] J. Bsaisou, J. de Vries, C. Hanhart, S. Liebig, U.-G. Meissner, D. Minossi et al., Nuclear Electric Dipole Moments in Chiral Effective Field Theory, JHEP 03 (2015) 104 [Erratum ibid. 1505 (2015) 083] [arXiv: 1411.5804] [INSPIRE].

[46] R.N. Mohapatra and G. Senjanović, Natural Suppression of Strong p and t Noninvariance, Phys. Lett. B 79 (1978) 283 [InSPIRE].

[47] A. Maiezza and M. Nemevšek, Strong P invariance, neutron electric dipole moment and minimal left-right parity at LHC, Phys. Rev. D 90 (2014) 095002 [arXiv:1407.3678] [INSPIRE].

[48] G. Senjanović and V. Tello, Restoration of Parity and the Right-Handed Analog of the CKM Matrix, arXiv:1502.05704 [INSPIRE].

[49] CMS collaboration, Search for heavy neutrinos and $\mathrm{W}$ bosons with right-handed couplings in proton-proton collisions at $\sqrt{s}=8 \mathrm{TeV}$, Eur. Phys. J. C 74 (2014) 3149 [arXiv:1407.3683] [INSPIRE].

[50] F.F. Deppisch, T.E. Gonzalo, S. Patra, N. Sahu and U. Sarkar, Signal of Right-Handed Charged Gauge Bosons at the LHC?, Phys. Rev. D 90 (2014) 053014 [arXiv:1407.5384] [INSPIRE].

[51] J.A. Aguilar-Saavedra and F.R. Joaquim, Closer look at the possible CMS signal of a new gauge boson, Phys. Rev. D 90 (2014) 115010 [arXiv:1408.2456] [INSPIRE].

[52] M. Heikinheimo, M. Raidal and C. Spethmann, Testing Right-Handed Currents at the LHC, Eur. Phys. J. C 74 (2014) 3107 [arXiv:1407.6908] [InSPIRE].

[53] J. Gluza and T. Jeliński, Heavy neutrinos and the $p p \rightarrow l l j j$ CMS data, Phys. Lett. B 748 (2015) 125 [arXiv: 1504.05568] [INSPIRE].

[54] F. Corriveau et al., Does The Positron From Muon Decay Have Transverse Polarization?, Phys. Lett. B 129 (1983) 260 [inSPIRE].

[55] H. Burkard et al., Muon Decay: Measurement of the Transverse Positron Polarization and General Analysis, Phys. Lett. B 160 (1985) 343 [InSPIRE]. 
[56] Zurich ETH-Cracow-PSI collaboration, K.U. Kohler, Determination of the polarization vector of positrons from the decay of polarized muons, hep-ex/0105021 [INSPIRE].

[57] W. Fetscher et al., Polarized muon decay: Measurement of the polarization vector of the decay positrons as a test of time reversal invariance, Nucl. Phys. A 721 (2003) 457 [INSPIRE].

[58] W. Fetscher et al., Measurement of the polarization vector of the $e^{+}$from the decay of polarized $\mu^{+}$as a test of time reversal invariance, J. Phys. G 29 (2003) 2017 [InSPIRE].

[59] R.A. Mann and M.E. Rose, Depolarization of Negative mu Mesons, Phys. Rev. 121 (1961) 293 [INSPIRE].

[60] V. Cirigliano, A. Kurylov, M.J. Ramsey-Musolf and P. Vogel, Lepton flavor violation without supersymmetry, Phys. Rev. D 70 (2004) 075007 [hep-ph/0404233] [INSPIRE].

[61] S. Bertolini, A. Maiezza and F. Nesti, Present and Future $K$ and B Meson Mixing Constraints on TeV Scale Left-Right Symmetry, Phys. Rev. D 89 (2014) 095028 [arXiv: 1403.7112] [INSPIRE].

[62] G. Senjanović and P. Senjanovic, Suppression of Higgs Strangeness Changing Neutral Currents in a Class of Gauge Theories, Phys. Rev. D 21 (1980) 3253 [InSPIRE].

[63] J. Chakrabortty, J. Gluza, R. Sevillano and R. Szafron, Left-Right Symmetry at LHC and Precise 1-Loop Low Energy Data, JHEP 07 (2012) 038 [arXiv:1204.0736] [INSPIRE].

[64] J. Chakrabortty, H.Z. Devi, S. Goswami and S. Patra, Neutrinoless double- $\beta$ decay in TeV scale Left-Right symmetric models, JHEP 08 (2012) 008 [arXiv:1204.2527] [INSPIRE].

[65] F.F. Deppisch, M. Hirsch and H. Pas, Neutrinoless Double Beta Decay and Physics Beyond the Standard Model, J. Phys. G 39 (2012) 124007 [arXiv:1208.0727] [INSPIRE].

[66] J. Barry and W. Rodejohann, Lepton number and flavour violation in TeV-scale left-right symmetric theories with large left-right mixing, JHEP 09 (2013) 153 [arXiv:1303.6324] [INSPIRE].

[67] C.-H. Lee, P.S. Bhupal Dev and R.N. Mohapatra, Natural TeV-scale left-right seesaw mechanism for neutrinos and experimental tests, Phys. Rev. D 88 (2013) 093010 [arXiv: 1309.0774] [INSPIRE].

[68] P. Bhupal Dev, S. Goswami and M. Mitra, TeV Scale Left-Right Symmetry and Large Mixing Effects in Neutrinoless Double Beta Decay, Phys. Rev. D 91 (2015) 113004 [arXiv: 1405.1399] [INSPIRE].

[69] T. Suzuki, D.F. Measday and J.P. Roalsvig, Total Nuclear Capture Rates for Negative Muons, Phys. Rev. C 35 (1987) 2212 [InSPIRE]. 